\title{
Storz Professional Image Enhancement System: A New Technique to Improve Endoscopic Bladder Imaging
}

Kamphuis GM¹, de Bruin DM ${ }^{1,2}$, Fallert $\mathrm{J}^{3}$, Gultekin $\mathrm{MH}^{4}$, de Reijke TM¹, Laguna Pes MP1 and de la Rosette JJMCH

${ }^{1}$ Department of Urology, Academic Medical Center, Amsterdam, Netherlands

${ }^{2}$ Department of Biomedical Engineering, Academic Medical Center, Amsterdam, Netherlands

3/maging Technologies Research, KARL STORZ Tuttlingen, Germany

${ }^{4}$ Cerrahpasa School of Medicine, Istanbul, Turkey

\begin{abstract}
Introduction: SPIES is a novel endoscopic imaging technique with different modalities. Chroma enhances the sharpness of the displayed image. Clara uses a local brightness adaptation in the image to achieve a clearer visibility of darker regions within the image. SPIES Spectra A and B are based on color tone shift algorithms to increase contrast.
\end{abstract}

Objectives: To describe the different SPIES modalities and to test them on bladder tissue images and tissue simulating phantoms with controlled optical properties.

Materials and methods: A bladder tumor image in both Chroma and Clara is analyzed on contrast related intensity fluctuations compared to the White Light image. To evaluate Spectra A and B, a validated tissue representing optical phantom model was used.

Results: Intensity fluctuations show the effect of the Chroma and Clara mode compared to White light. The SPIES A and B modalities change the effective spectral response in the imaging system. This was shown and measured in the phantom model by an increased absorbance for the superficial layers (Spectra A) and the deeper layers (Spectra B).

Conclusion: All SPIES modalities show visual and quantitative differences, expressed as increased image intensity or pixel-to-pixel intensity difference (Chroma and Clara) or increased contrast (Spectra A and B).

Keywords: SPIES; Endoscopy; Cystoscopy; Image enhancement

\section{Abbreviations}

NMIBC: Non-Muscle Invasive Bladder Cancer; TURBT: Transurethral Resection of a Bladder Tumor; PDD: Photodynamic Diagnosis; WL: White Light; NBI: Narrow Band Imaging; SPIES: Storz Professional Image Enhancement; nm: Nanometer; SA: Spectra A; SB: Spectra B; $\mu \mathrm{m}$ : Micrometer; CROES: Clinical Research Office of the Endourological Society; EAU: European Association of Urology

\section{Introduction}

Non-muscle invasive bladder cancer (NMIBC) has a high incidence and due to its high recurrence rate, close follow-up is warranted. Although patients with NMIBC have a relatively good prognosis in terms of cancer-specific survival, the chance that the cancer will recur has been estimated to be as high as 75\% [1]. Several studies reported that the bladder cancer recurrence rate at first follow-up cystoscopy at 3 months after transurethral resection of a bladder tumour (TURBT) is up to $45 \%$ [2]. It remains unknown if this is already a recurrence, or an overlooked tumour, or tumour persistence. However, it has been estimated that $10-20 \%$ of bladder tumors are overlooked in conventional white light (WL) cystoscopy [3].

Therefore, novel endoscopic imaging techniques have been introduced and tested to improve the detection of bladder cancer e.g. photodynamic diagnosis (PDD) using HEXVIX, and narrow band imaging (NBI). These techniques have shown to reduce the number of interventions and reduce costs [4].

Karl Storz has recently developed the SPIES system. SPIES are an acronym for Storz Professional Image Enhancement System. This system offers several image enhancement modalities that can be used in combination with SPIES adapted camera heads and video endoscopes. We describe for the first time the different SPIES modalities: Spectra A, Spectra B, Chroma and Clara in the field of bladder tumour. It is hypothesized that the SPIES modalities will enhance the images obtained during endoscopy enabling a better bladder imaging and thus better detection of bladder tumours.

In the current release (Image 1 Connects [TC200] and Image 1 H3-Link [TC300]) (Storz, Tuttlingen, Germany) five SPIES modalities, besides a conventional WL image, are available: Chroma, Clara, Clara and Chroma combined, Spectra A and Spectra B. The Chroma modality should enhance the sharpness of the image. The Clara modality is designed to create a clearer image of darker regions within the image. Together they should provide a clearer and sharper image of the original WL image. The Spectra A and Spectra B modality change the effective spectral response in the imaging system, which should lead to a better color contrast (Figure 1). Furthermore, the system is capable

*Corresponding author: Kamphuis GM, Department of Urology, Academic Medical Center, Amsterdam, Netherlands, Tel: +31 205661453; Fax: +31 20 5669585; E-mail: g.m.kamphuis@amc.uva.nl

Received February11, 2016; Accepted March 25, 2016; Published March 28, 2016

Citation: Kamphuis GM, de Bruin DM, Fallert J, Gultekin MH, de Reijke TM, et al. (2016) Storz Professional Image Enhancement System: A New Technique to Improve Endoscopic Bladder Imaging. J Cancer Sci Ther 8: 071-077. doi:10.4172/1948-5956.1000394

Copyright: (c) 2016 Kamphuis GM, et al. This is an open-access article distributed under the terms of the Creative Commons Attribution License, which permits unrestricted use, distribution, and reproduction in any medium, provided the original author and source are credited. 


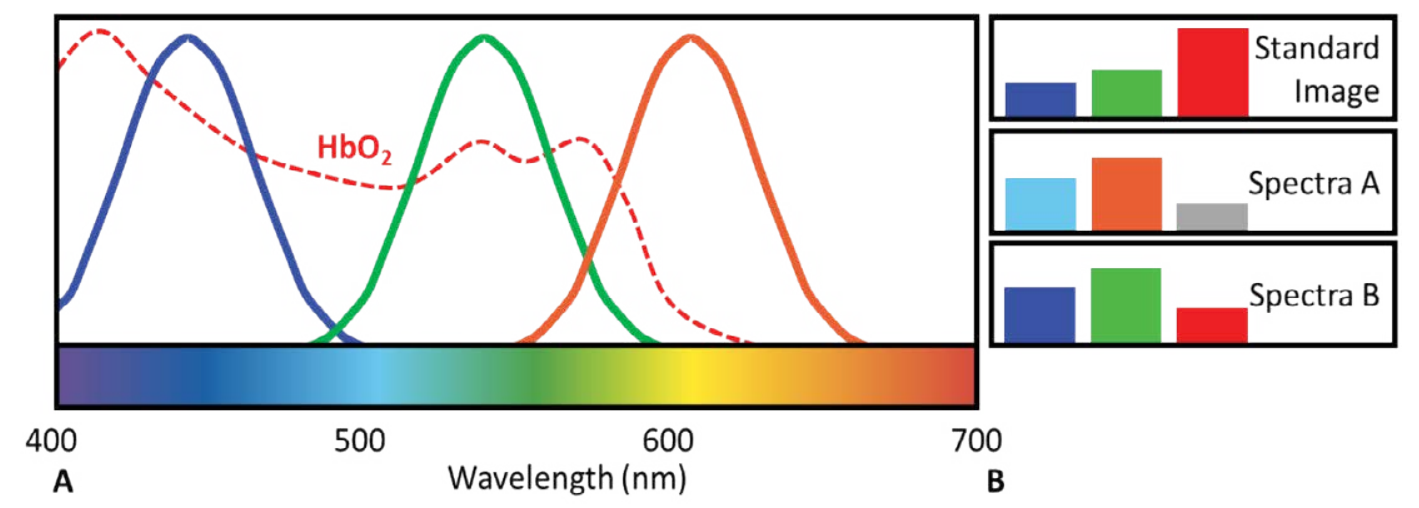

Figure 1: A Left side: Schematic of the camera response with respect to the oxygenated haemoglobin absorption curve. B Right side: Schematic explanation of the specific colour renderings in the SPIES Spectra modes for such a typical spectrum.

to provide a standard WL image in a side-by-side viewing mode with the SPIES image simultaneously.

\section{SPIES chroma}

The SPIES Chroma mode is designed to enhance the sharpness of the displayed image. The global color rendering of the image is identical to the standard WL image, but the local contrast of present color differences is to be enhanced. The core computation of this mode is based on an un-sharp masking algorithm, where the processed image is given by the original image plus a scaled difference between the original and its Gaussian filtered output [5]. To achieve this, SPIES Chroma analysis for each image pixel a large surrounding area according to the present spatial color changes. To do this processing in real-time requires a much higher computational effort compared to classical enhancement algorithms, which are e.g. only capable to change the brightness step at the edges of a vessel instead of enhancing the visibility of the whole vessel as done by SPIES Chroma. By using SPIES Chroma the goal is to get a better visibility of structural tissue information and typical disturbing black rings around reflections that are common for classical edge enhancement are meant to be avoided. An example of an image in SPIES Clara next to a WL image is shown in Figure 2.

\section{SPIES clara}

The SPIES Clara mode is designed to use a local brightness adaptation in the image to achieve a clearer visibility of darker regions within the image. The goal is that more can be seen in the dark region and the viewing comfort for the user is increased. An example of an image in SPIES Clara next to a WL image is shown in Figure 3. Optionally, this mode can also be used in combination with the contrast enhancement provided in the Chroma algorithm. This combination is then accessible by a distinct SPIES Clara + Chroma mode.

\section{SPIES spectral modes}

Two different predefined modes for virtual chromo endoscopy are available in the current device, denominated as SPIES Spectra A and SPIES Spectra B. These modes change the effective spectral response in the imaging system, so that a different color contrast can be observed on the video screen.

SPIES spectra A: The SPIES Spectra A mode is based mainly on the green $(\sim 500-570 \mathrm{~nm})$ and blue $(\sim 400-480 \mathrm{~nm})$ light spectral signals that are separated in the RGB response of the camera system (Figure
1). In these bands the hemoglobin absorption is significantly higher compared to the red spectral band above $570 \mathrm{~nm}$. Since the absorption coefficient $\mu_{\mathrm{a}}$ is the main limiting parameter of the light penetration depth $\delta$ (see equation 1), the blue and green spectral parts are limited to a short to mid-range tissue-penetration depth in the order of one millimeter.

$$
\delta=\frac{1}{\mu_{e f f}}=\frac{1}{\sqrt{3 \mu_{a}\left(\mu_{a}+\mu_{s}^{\prime}\right.}}
$$

Equation 1: Light penetration depth $\delta$ dependency on reduced scattering coefficient $\mu_{\mathrm{s}}$ and absorption coefficient $\mu_{\mathrm{a}}$ in diffusion approximation [6].

A dedicated color transformation pronounces the contrast within these two spectral bands by changing the perceived output color with respect to the original colors created by this spectral input. Due to the limited penetration depth in the blue to green spectral part, this mode allows to highlight the contrast of capillaries and vessels in the superficial mucosa and sub mucosa (Figure 4).

SPIES spectra B: The SPIES Spectra B pronounces as well as the Spectra A mode the blue to green spectral part in order to reach a higher contrast in the superficial mucosa and sub mucosa. Differently, this mode is based on a color tone shift algorithm to reduce the dominant diffuse red spectral reflection, while still keeping the whole spectral information contained in the original color bands [7]. Thus, in addition to the blue to green spectral band the contrast of capillaries and vessels are highlighted without losing the additional information from deeper tissue layers that are only visible in the red spectral part (Figure 5).

\section{Objectives}

We hypothesize that SPIES Chroma enhances sharpness of the image. SPIES Clara enhances local brightness, Spectra A (SA) and Spectra B (SB), which both use specific colour renderings to pronounce the spectral separation of the recorded broad visible spectrum, will increase contrast of a bladder lesion. The objective of this study is to describe the different SPIES modalities and to test the image enhancement capabilities of the SPIES image modalities, SPIES Chroma and SPIES Clara, Spectra A and Spectra B, on bladder tissue images and optical phantoms with controlled optical properties.

\section{Material and Methods}

To investigate the difference between the white light and Clara mode, an image in both settings is analyzed on intensity differences 
Citation: Kamphuis GM, de Bruin DM, Fallert J, Gultekin MH, de Reijke TM, et al. (2016) Storz Professional Image Enhancement System: A New Technique to Improve Endoscopic Bladder Imaging. J Cancer Sci Ther 8: 071-077. doi:10.4172/1948-5956.1000394

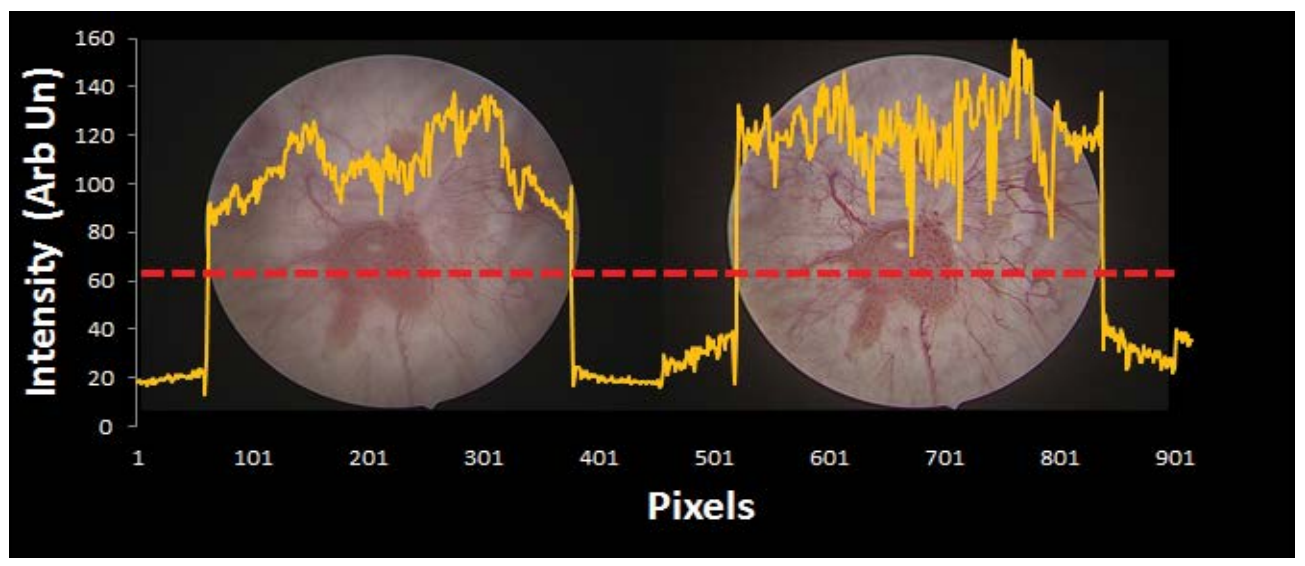

Figure 2: Example during a cystoscopy using the SPIES Chroma mode (right) in a side-by-side dual view with the unprocessed white light image (left). The light intensity, plotted as the yellow overlay in the image is measured at the red line location in the image. Sharp demarcations are clearly visible in the right image as well as in the plotted graph that shows larger fluctuations on the right side caused by larger contrast variations.

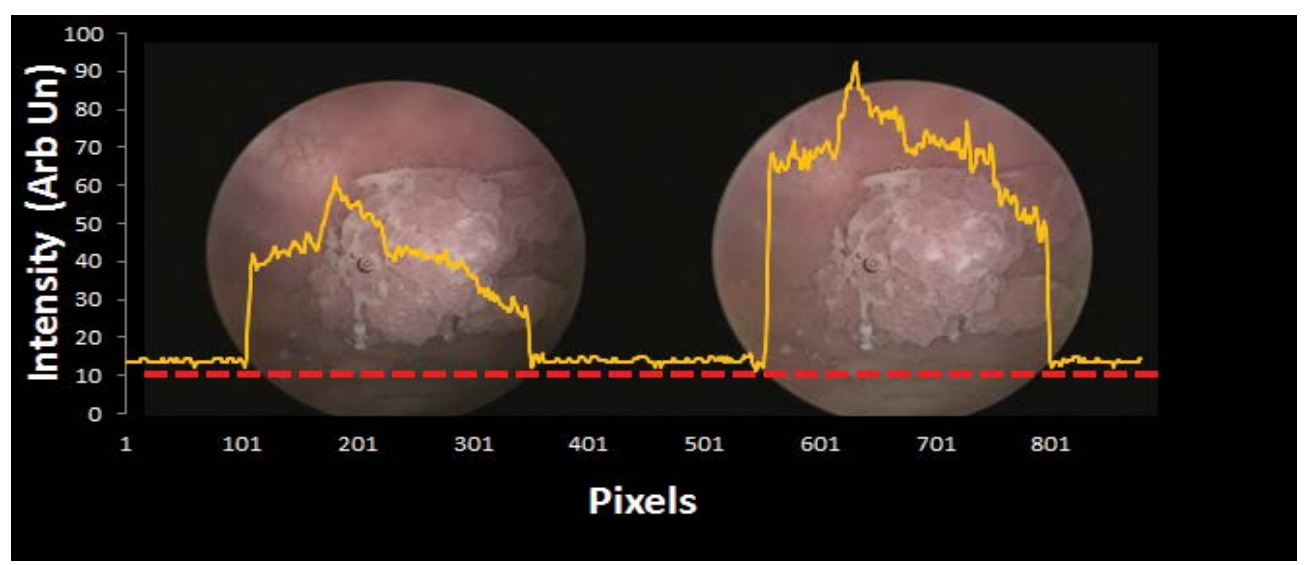

Figure 3: Example during a cystoscopy using the SPIES Clara mode (right) in a side by side dual view with the unprocessed white light image (left). The light intensity, plotted as the yellow overlay in the image is measured at the red line location in the image. Increased intensity is clearly visible in the right image as shown in the right plotted graph with an intensity increase in Clara mode of $\sim 30$ counts (Arb Un).

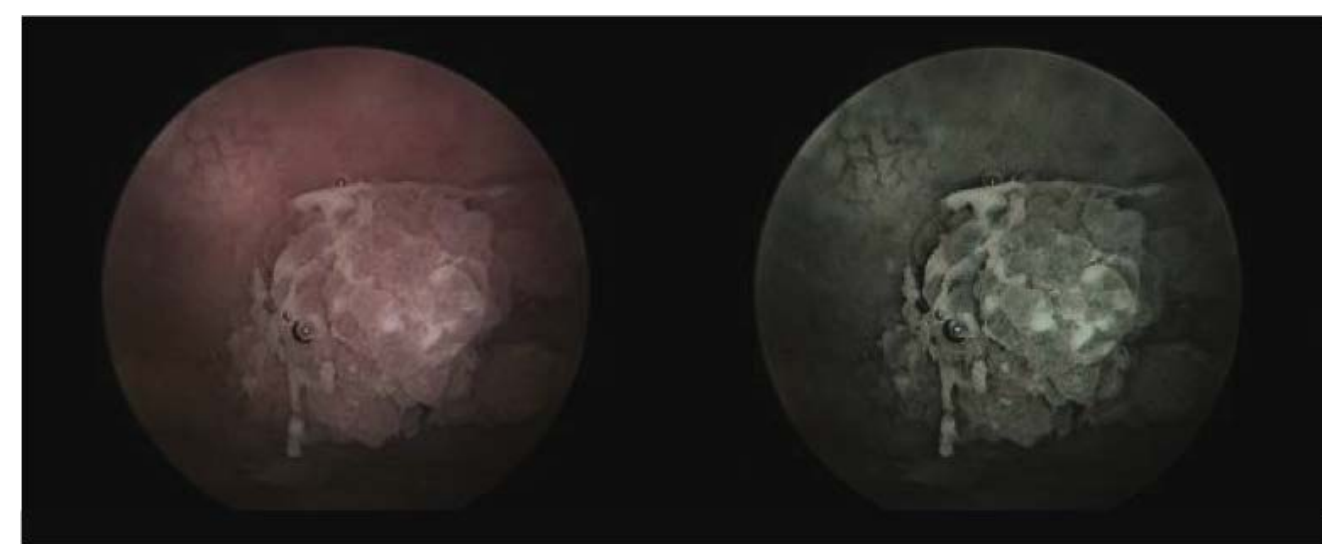

Figure 4: Example during a cystoscopy using the SPIES Spectra A mode (right) in a side-by-side dual view with the unprocessed image (left).

between dark and bright regions. The light intensity is measured at a horizontal line (dashed red line) vs. the pixels and shown as an overlay over the image as shown in Figure 3B. To analyze the difference between the white light and Chroma mode, an image in both settings is analyzed on contrast related intensity fluctuations. The light intensity is measured at a horizontal line (dashed red line) vs. the pixels and shown as an overlay over the image as shown in Figure 2B. 
Citation: Kamphuis GM, de Bruin DM, Fallert J, Gultekin MH, de Reijke TM, et al. (2016) Storz Professional Image Enhancement System: A New Technique to Improve Endoscopic Bladder Imaging. J Cancer Sci Ther 8: 071-077. doi:10.4172/1948-5956.1000394

To evaluate the spectral properties of the SPIES system, we have tested the two spectral modes on a validated optical phantom model. Figure 6 gives a schematic representation of the phantom experiment.

A capillary, filled with human blood, was placed under three gradually stacked $\mathrm{TiO}_{2}$-silicone phantoms of $100 \mu \mathrm{m}$ each. Each phantom contained a 0.2 weight $\% \mathrm{TiO}_{2}$ concentration representing a scattering value of $4 \mathrm{~mm}^{-1}$ at $700 \mathrm{~nm}$ as described by de Bruin et al. [8]. The whole phantom-capillary stack was illuminated and imaged with the SPIES endoscope. The SPIES endoscope was placed $5 \mathrm{~cm}$ above the stack for each measurement. The resulted image shows an image with increased layer thickness. This allows measurement of contrast differences caused by the absorbance of light for different depths. Within the acquired image, a region of interest (ROI) was defined which includes the blood capillary which was used as the intensity measurement (I). This was compared to a light intensity $\left(\mathrm{I}_{0}\right)$ measurement of an identical ROI directly next to the capillary and displayed as percentage of absorbance using:

$$
\text { Absorbance }(\%)=1-\frac{I}{I_{0}} \times 100
$$

Statistical testing between WL, SA and SB within each imaging depth location was performed using a simple paired student T-test (Medcalc version 14.8.1). The error bars in Figures 7-9 represents the standard deviation of the normalized intensity profile within a region of interest at each depth location.

\section{Results}

\section{SPIES chroma}

To address the difference between normal WL endoscopy and the SPIES Chroma mode, the data was analysed on intensity differences within both images. These differences are depicted in Figure 2. Figure $2 \mathrm{~A}$ shows an illustration to explain the effect of Chroma. Figure $2 \mathrm{~B}$ gives an actual example of the SPIES Chroma mode next to a conventional WL image during a cystoscopy. The light intensity, plotted as the yellow overlay in the image is measured at the red line location in the image to show the Chroma effect compared to the WL. A clear difference is observed in intensity fluctuations between the WL imaging ( 5\%) and Chroma imaging ( 25\%). These numbers reflect the visual impression of increased contrast and sharpness of the image.

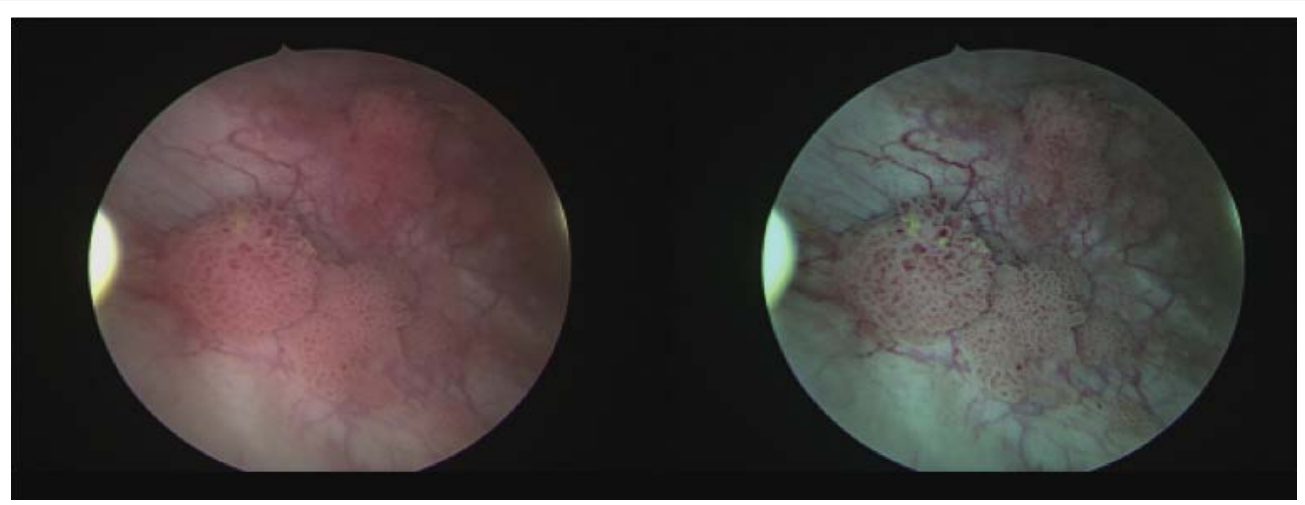

Figure 5: Example during a cystoscopy using the SPIES Spectra B mode (right) in a side-by-side dual view with the unprocessed white light image (left)

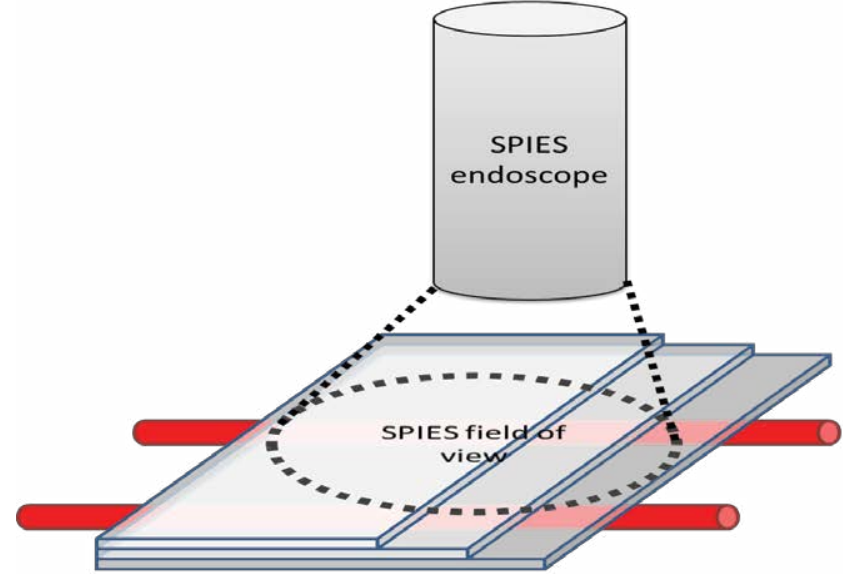

A

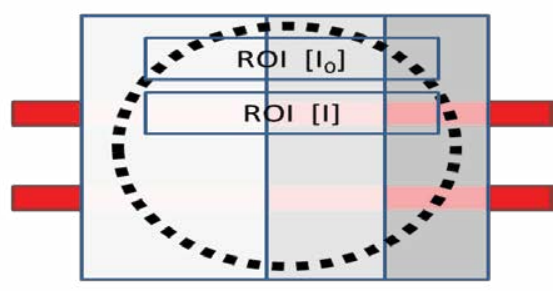

B

Figure 6: A, Schematic representation of the phantom experiment. A capillary, filled with human blood, was placed under three stair-wise stacked TiO2silicone phantoms of $100 \mu \mathrm{m}$ each. Each phantom contained a $0.2 \% \mathrm{TiO}_{2}$ concentration representing a scattering value of $4 \mathrm{~mm}^{-1}$ at $700 \mathrm{~nm}^{\text {. The whole }}$ phantom-capillary stack was illuminated with the SPIES endoscope. The endoscope was placed $5 \mathrm{~cm}$ above the stack. The resulted image shows an enface image with increased layer thickness as schematically shown in $6 \mathrm{~B}$. This allows measurement of contrast differences caused by absorbance for different depths. 
Citation: Kamphuis GM, de Bruin DM, Fallert J, Gultekin MH, de Reijke TM, et al. (2016) Storz Professional Image Enhancement System: A New Technique to Improve Endoscopic Bladder Imaging. J Cancer Sci Ther 8: 071-077. doi:10.4172/1948-5956.1000394

\section{SPIES clara}

To address the difference between normal WL endoscopy and the SPIES Clara mode, the data was analyzed on intensity differences within both images. These differences are depicted in Figure 3. Figure $3 \mathrm{~A}$ shows an illustration to explain the effect of Clara. Figure 3B gives an actual example of the SPIES Clara mode next to a conventional WL image during a cystoscopy. The light intensity, plotted as the yellow overlay in the image is measured at the red line location in the image to show the Clara effect compared to the WL. A clear difference is observed in intensity offset, ranging from 40 intensity counts (Arb Un, white light) to 70 (Arb Un, Clara) This indicates an increased intensity in the darker areas of the image.

\section{SPIES spectra A}

The effects on image contrast of spectra A mode were validated in a multilayer phantom model. For each phantom layer thickness, the absorbance was measured. The absorbance of light was $14 \% \pm 2 \%$ for the $100 \mu \mathrm{m}$ layer thicknesses, $10 \% \pm 2 \%$ for the $200 \mu \mathrm{m}$ layer thickness and $1 \% \pm 2 \%$ for the $300 \mu \mathrm{m}$ layer thickness (Figure 7). Figure 4 depicts an exemplary image from a papillary lesion obtained with white light and Spectra A simultaneously.

\section{SPIES spectra $B$}

The effects on image contrast of spectra B mode were validated in a multilayer phantom model. For each phantom layer thickness, the absorbance was measured. The absorbance of light was $14 \% \pm 2 \%$ for the $100 \mu \mathrm{m}$ layer thicknesses, $11 \% \pm 2 \%$ for the $200 \mu \mathrm{m}$ layer thickness and $4 \% \pm 2 \%$ for the $300 \mu \mathrm{m}$ layer thickness (Figure 8 ). Figure 5 depicts an exemplary image from a papillary lesion obtained with white light and Spectra B simultaneously.

\section{SPIES Spectra A and Spectra B vs White light}

Figure 9 demonstrates a comparison of absorbance (\%) for white light, spectra A and spectra B mode tested for a 100, 200 and $300 \mu \mathrm{m}$ thick scattering layer above a blood filled capillary.

For the superficial layer $(100 \mu \mathrm{m}) \mathrm{WL}$ shows a mean absorbance of $12.0 \pm 1.8 \%$ while both spectra A and B show an increasing absorbance of $13.6 \pm 1.7 \%$ and $13.7 \pm 1.9 \%$, respectively. For the intermediate layer
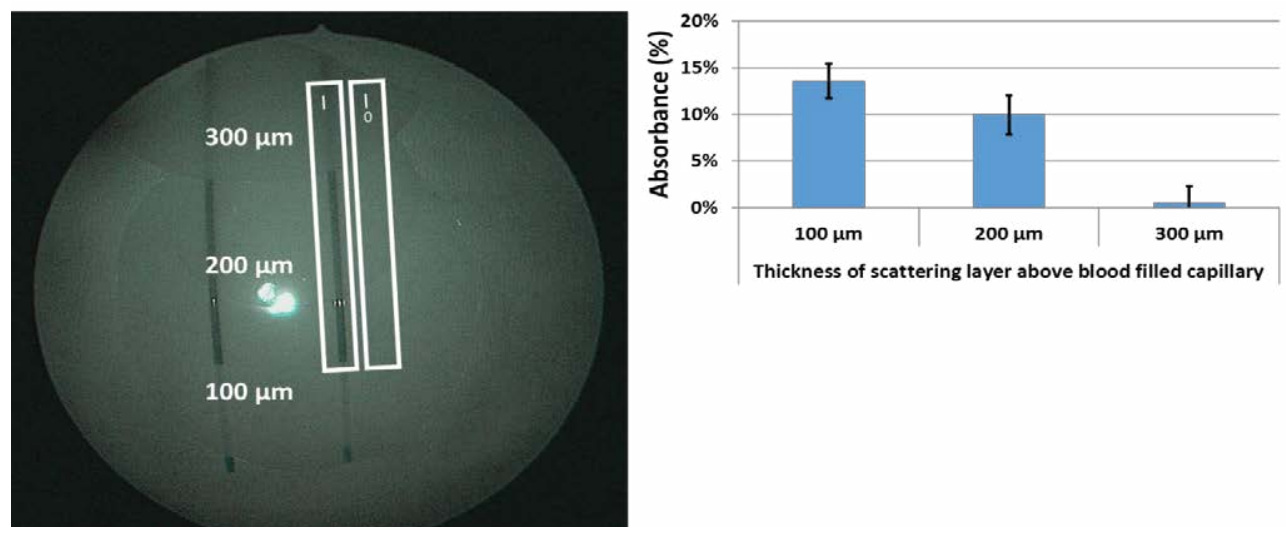

Figure 7: Phantom experiment in spectra A mode. For each thickness, the absorbance was measured. The absorbance of light was $14 \% \pm 2 \%$ for the $100 \mu m$ layer thickness, $10 \% \pm 2 \%$ for the $200 \mu \mathrm{m}$ layer thickness and $1 \% \pm 2 \%$ for the $300 \mu \mathrm{m}$ layer thickness. The error bars in figure 7 represents the standard deviation of the normalized intensity profile within a region of interest at each depth location.
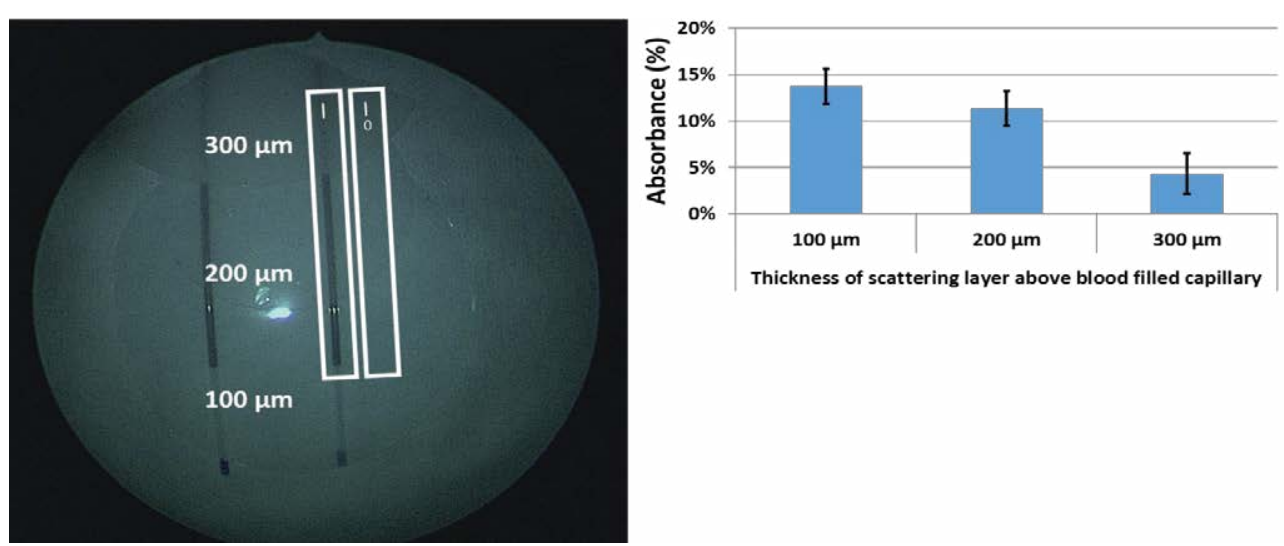

Figure 8: Phantom experiment in spectra B mode. For each thickness, the absorbance was measured. The absorbance of light was $14 \% \pm 2 \%$ for the $100 \mu \mathrm{m}$ layer thickness, $11 \% \pm 2 \%$ for the $200 \mu \mathrm{m}$ layer thickness and $4 \% \pm 2 \%$ for the $300 \mu \mathrm{m}$ layer thickness. The error bars in Figure 8 represents the standard deviation of the normalized intensity profile within a region of interest at each depth location. 


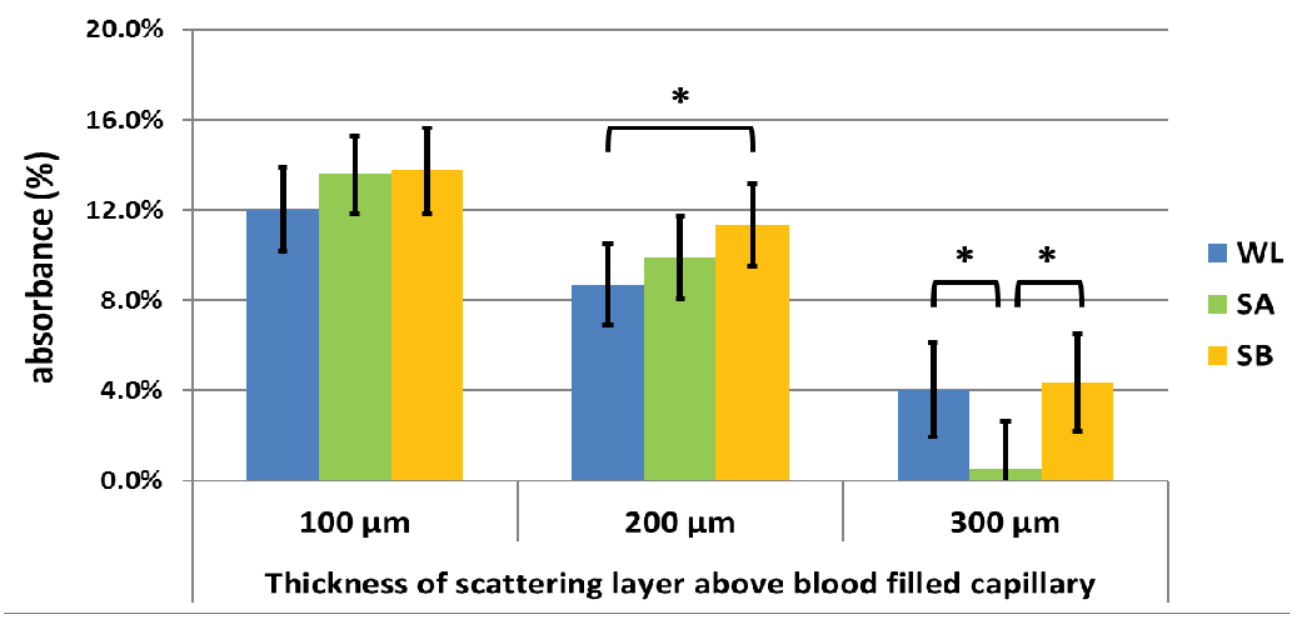

Figure 9: Comparison of absorbance (\%) for white light, spectra A and spectra B modes tested for a 100,200 and $300 \mu \mathrm{m}$ thick scattering layer above a blood filled capillary. Significant differences are indicated with a * The error bars in Figure 9 represents the standard deviation of the normalized intensity profile within a region of interest at each depth location. For explanation see paragraph 'SPIES Spectra A and B vs White light'.

$(200 \mu \mathrm{m})$, all modalities show overall a lower absorbance compared to the $100 \mu \mathrm{m}$ layer. The WL shows a mean absorbance of $8.7 \pm 1.8 \%$ while both spectra A and B show a higher absorbance of $9.9 \pm 1.8 \%$ and 11.3 $\pm 1.9 \%$, respectively. This demonstrates that spectra $\mathrm{B}$ is more sensitive for absorbance in the intermediate layers compared to WL $(p=0.001)$. For the thickest layer $(300 \mu \mathrm{m})$ all measured absorbance are lower. The WL shows a mean absorbance of $4.0 \pm 2.1 \%$ while both spectra A and B show a mean absorbance of $0.5 \pm 2.1 \%$ and $4.4 \pm 2.2 \%$, respectively. This demonstrates that WL and Spectra B are both more sensitive for absorbance of deeper located blood vessels when compared to the spectra A setting $(\mathrm{p}=0.001)$.

\section{Discussion}

In the field of evaluation of quality of endoscopic images much is still unknown. The theoretical benefits of the SPIES system are that with Clara and Chroma, a better and sharper image is obtained that will improve visualization and interpretation and thus tumour detection and treatment. The Spectra A and B might improve the evaluation of images by changing the colour response and thus the contrast that would help in differentiation of normal mucosa and tumour. To objectify the spectral SPIES modalities, a phantom model was used and absorbance was measured. For the other modalities, pixel intensities were plotted to show the difference with unprocessed images. Based on these findings, we have concluded that the new modalities are indeed different. However, it should be noted that this does not necessarily mean that the modalities are an improvement for the diagnosis of disease.

Recent developments in endoscopic techniques aim to improve our capability to detect and thus treat cancer in an optimal way. The first goal in bladder cancer treatment is to improve visualization, which will result in a more complete endoscopic resection that possibly reduces adjuvant treatments and subsequently reduces cancer recurrence and progression $[9,10]$.

Different techniques are nowadays available to improve endoscopic imaging and detection of bladder tumours. To summarize, PDD uses the biochemical response of urothelial cancers resulting in better detection. NBI uses a narrow band light to highlight contrast between different vascularized mucosal areas. And SPIES contains five modalities that change the endoscopic image by increasing sharpness, increasing light in dark areas and increasing contrast.

A systematic review and meta-analysis of bladder tumour detection demonstrated a higher sensitivity for PDD compared with WL cystoscopy [11]. A large, multicentre, prospective randomized trial comparing HAL fluorescence-guided TURB with standard TURB reported an absolute recurrence rate reduction of $9 \%$ within 9 months [12]. The EAU guidelines state that the use of fluorescence-guided biopsy and resection are more sensitive than conventional procedures for detection of bladder cancer, particularly for CIS [13]. The drawback is that PDD has a lower specificity than white light endoscopy $(63 \%$ versus $81 \%$ ). False positivity can be induced by inflammation or recent TUR, and during the first three months after BCG instillation [13]. Other disadvantages of the use of PDD are the intravesical instillation 2 to 4 hours before cystoscopy and extra equipment necessary, which leads to more complex organization, increased costs and patient's discomfort.

A systematic review and meta-analysis demonstrated that NBI is an effective method for the identification of abnormal lesions including CIS [4]. The specificity of NBI cystoscopy was lower compared to WL cystoscopy which was explained by possible inflammatory lesions after intravesical therapy [4]. The Clinical Research Office of the endourological society (CROES) recently finished recruitment of a randomized controlled trial (RCT) comparing WL and NBI supported TURBT that should help define the role of NBI in preventing recurrences. The use of NBI has a level of evidence 3 according to the recent EAU Guidelines [13].

SPIES aim to enhance endoscopic imaging and thus improve tumor detection and treatment. The current release contains besides a conventional white light image five modalities; Chroma, Clara, Chroma and Clara, Spectra A and Spectra B. We have tested the Chroma and Clara system and found a clear increase on sharpness and brightness. As well in the cystoscopy images subjectively, as measured by light intensity on per procedure taken images.

To objectivize the contrast effect of Spectra A and B we performed a phantom test. We took images to compare light absorbance under three different layer thicknesses of 100, 200 and $300 \mu \mathrm{m}$. As 
hypothesized, there is a difference between Spectra A and B because of specific colour renderings that are used to pronounce the spectral differentiation, because light penetration in tissue is depending on wavelength. As shown in Figure 9 there is more absorbance of light in 100 and $200 \mu \mathrm{m}$ tissue thicknesses, thus the contrast is higher. While Spectra A concentrates on the absorbance of the superficial layers only, Spectra B is also sensitive to absorbance in deeper layers.

The hypothesis is that a clearer and sharper image and more contrast lead to a higher chance to distinguish differences in tissues and thus have a higher detection rate of abnormalities e.g., Tumours. There is today no clinical data to support or reject these hypothesized benefits of SPIES.

To evaluate the perception of the different SPIES modalities the íSPIES study has been conducted. In this study 4 images of the same area in the bladder was caught in different SPIES modalities. The inspiration for this study was found in the field of radio-oncology, where differences in delineation for the field of radiotherapy by different participants were studied [14]. In total 20 areas were caught in 4 image modalities. These 80 images $(+20$ control images) were judged on image quality by 73 urologist) were judged on image quality by 73 urologist and they and they delineated the area(s) they found suspicious for possible malignancy. In easy to evaluate cases, there was no difference found in the delineated area between images in the different modalities. In the more difficult to evaluate cases however, images in CC and SB have less variation in interpretation than WL and SA. The image quality in SPIES modalities was graded significantly higher than in WL [15].

Besides this evaluation, the Clinical Research Office of the CROES has launched a multi-centre randomized clinical trial to compare SPIES TURBT with White light TURBT in the end of 2014 which is enrolling patients today [16].

\section{Conclusions}

In this manuscript the new storz professional image enhancement system (SPIES) is explained, a phantom test of the different modalities for its abilities is described and cystoscopic differences of bladder tumours are shown. The SPIES Chroma modality uses local contrast of present colour differences to enhance the sharpness of the image. The Clara mode uses a local brightness adaption in the image to achieve a clearer visibility of darker regions within the image. Together they provide a clearer and sharper image of the original white light image. The SPIES A and B modality change the effective spectral response in the imaging system, so that a better color contrast can be observed. This contrast effect is depending on the tissue layer thickness.

\section{References}

1. Sylvester RJ, van der Meijden AP, Oosterlinck W, Witjes JA, Bouffioux C, et al (2006) Predicting recurrence and progression in individual patients with stage Ta T1 bladder cancer using EORTC risk tables: a combined analysis of 2596 patients from seven EORTC trials. Eur Urol 49: 475-477.

2. Brausi M, Collette L, Kurth K, van der Meijden AP, Oosterlinck W, et al. (2002) Variability in the recurrence rate at first follow-up cystoscopy after TUR in stage Ta T1 transitional cell carcinoma of the bladder : a combined analysis of seven EORTC studies. Eur Urol 41: 523-531.

3. Jichlinski $P$ and Leisinger HJ (2005) Fluorescence cystoscopy in the management of bladder cancer: a help for the urologist! Urol Int 74: 97-101.

4. Zheng C, Lv Y, Zhong Q, Wang R, Jiang Q (2012) Narrow band imaging diagnosis of bladder cancer: systematic review and meta-analysis. BJU Int 110: E680-687.

5. Ramponi G, Strobel N, Mitra SK, Yu TH (1996) Nonlinear unsharp masking methods for image contrast enhancement. Journal of Electronic Imaging 5: 353-366.

6. Wang LV and Wu H (2007) Biomedical Optics: Principles and Imaging. John Wiley \& Sons

7. Sid Ahmed MA (1995) Image Processing: Theory, Algorithms, and Architectures. McGraw-Hill.

8. de Bruin DM, Bremmer RH, Kodach VM, de Kinkelder R, van Marle J, et al. (2014) Optical phantoms of varying geometry based on thin building blocks with controlled optical properties. J Biomed Opt 15: 025001

9. Liu JJ, Droller MJ, Liao JC (2012) New optical imaging technologies for bladder cancer: considerations and perspectives. J Urol 188: 361-368.

10. Lopez A, Liao JC (2014) Emerging endoscopic imaging technologies for bladder cancer detection. Curr Urol Rep 15: 406.

11. Mowatt G, N'Dow J, Vale L, Nabi G, Boachie C, et al. (2011) Photodynamic diagnosis of bladder cancer compared with white light cystoscopy: Systematic review and meta-analysis. Int J Technol Assess Health Care 27: 3-10.

12. Stenzl A, Penkoff H, Dajc-Sommerer E, Zumbraegel A, Hoeltl L, et al. (2011) Detection and clinical outcome of urinary bladder cancer with 5-aminolevulinic acid-induced fluorescence cystoscopy: A multicenter randomized, doubleblind, placebo-controlled trial. Cancer 117: 938-47.

13. Babjuk M, Burger M, Zigeuner R, Shariat SF, van Rhijn BW, et al. (2013) EAU guidelines on non-muscle-invasive urothelial carcinoma of the bladder: update 2013. Eur Urol 64: 639-53.

14. Steenbakkers RJ, Duppen JC, Fitton I, Deurloo KE, Zijp L, et al. (2005) Observer variation in target volume delineation of lung cancer related to radiation oncologist-computer interaction: a "big brother"evaluation. Radiother Oncol 77: 182-190

15. Kamphuis GM, de Bruin DM, Brandt MJ, Knoll T, Conort P, et al. (2016) Comparing image perception of bladder tumours in four different storz professional image enhancement system SPIES modalities using the íSPIES app. J Endourol.

16. Gravas S and Stenzl A (2014) News from Clinical Research Office of the Endourological Society (CROES). J. Endourol. 25: 1399-1402. 